\title{
The current status of survivorship care provision at the state level: a Wisconsin-based assessment
}

\author{
Alexandria L. Cull Weatherer ${ }^{1}$ (D) John K. Krebsbach ${ }^{1} \cdot$ Amye J. Tevaarwerk $^{1,2} \cdot$ Sarah C. Kerch $^{1} \cdot$ Noelle K. LoConte $^{1,2}$
}

Received: 9 June 2021 / Accepted: 23 September 2021 / Published online: 5 October 2021

(c) The Author(s), under exclusive licence to Springer Science+Business Media, LLC, part of Springer Nature 2021

\begin{abstract}
Purpose As the number of cancer survivors grows, the responsibility for addressing their unique physical and emotional needs also increases. Survivorship care services vary by geography, health system, and insurance coverage. We aimed to understand the state of survivorship care services in Wisconsin's cancer facilities.

Methods The selection of cancer treatment facilities sought to provide a geographically representative sample. An adapted Patient-Centered Survivorship Care Index was comprised of questions regarding different aspects of survivorship practices. Areas of interest included disciplines incorporated, services provided, standards of care, and discussion of late-term effects, among others.

Results Out of 90 sites invited, 40 responded (44.4\%). Oncologists, physician assistants, and nurse practitioners were the most common follow-up care disciplines. Risk reduction services, dietary services, access to physical activity, and behavioral health specialist referral were described as standards of care in less than half of sites. All sites reported working with community partners, $92.5 \%$ of which worked with YMCA-related programs. Discussion of long-term effects was a standard of care for all sites. Effects such as emotional distress and health practice changes were frequently discussed with almost all patients, while sexual functioning and fertility were not.

Conclusions Services and specialties related to behavioral health, fertility/sexual health, and rehabilitation and physical activity varied between sites. Such services may be offered less often due to variable insurance coverage.

Implications for Cancer Survivors Policy solutions should be explored to increase insurance coverage and provision rates of necessary survivorship services to keep up with the projected increase in demand. Given imperfect and evolving measurement tools to assess needs for cancer survivorship care services, cancer survivors should feel empowered to voice when they have unmet needs and request referrals.
\end{abstract}

Keyword Cancer survivorship; Health Care delivery; Exercise; Sexual health; Psychological; Quality of life

\section{Background}

The National Cancer Institute (NCI) defines survivorship as the overall health and well-being of a person with cancer from the time of initial diagnosis through the end of life [1]. Nearly 17 million Americans had been diagnosed or were living with cancer at the start of 2019, a number that is projected to grow to over 22 million by the beginning

Alexandria L. Cull Weatherer

acull@wisc.edu

1 University of Wisconsin Carbone Cancer Center, $610 \mathrm{~N}$ Walnut St., Room 370 WARF, Madison, WI 53726, USA

2 Department of Medicine, School of Medicine and Public Health, University of Wisconsin, Madison, WI, USA of 2030 [2,3]. Current literature also shows most cancer survivors are older than 65 years old $[2,4]$. With older age comes an increase in severe comorbidities and more complicated healthcare needs [3]. Such trends exacerbate the detrimental effects of cancer diagnosis and treatment on health and quality of life [5,6]. The fragmented healthcare system and an over-dependence on the oncologist to provide care coordination, in addition to treatment, may prove unsustainable given the predicted oncology provider shortage [7]. Considering the growth in the survivor population and rising rates of some adolescent and young adult (AYA) cancers, it is important to acknowledge and prioritize cancer survivors' unique needs, and the variety of services needed to meet these needs, while also addressing the unsustainability of the existing cancer care delivery model [8-10]. 
The development and implementation of multi-disciplinary cancer survivorship programs is one way to meet the needs of a growing population of cancer survivors [11].

Considering the growing demand and limited evidence for an effective care model, survivorship care standards and models in the last ten years have evolved rapidly as various approaches are further studied $[2,8]$. Consequently, significant variability in the provision and quality of survivorship care has been noted $[8,11]$. Cancer survivorship programs are usually only found in large cancer centers due to cost and poor reimbursement [12]. Gaps in survivorship care include poor integration of survivorship care transitions from oncology to primary care, lack of knowledge regarding cancer survivor needs, differing attitudes and perceptions of ideal survivorship care models and financial barriers for providing and receiving survivorship services [2, 8]. Limited literature shows that long-term cancer survivors face higher costs of care, trouble accessing care, and issues maintaining insurance coverage, which is associated with worse access to and receipt of cancer care, and lower survival [2, 13-15]. Few studies have explored disparities that may persist into posttreatment survivorship or the impact of insurance on the availability of survivorship services and utilization, especially for rural survivors $[14,16,17]$. Survivors in rural areas face reduced access to oncology services and multidisciplinary providers, limited clinical trial opportunities, travel barriers, and higher rates of cancer-related mortality [17]. Furthermore, other disparities are noted for survival, resection receipt, and treatment receipt along racial and ethnic lines $[2,14,15,18]$. More research is needed to identify the variables that may impact and increase survivorship service utilization.

Wisconsin offers a unique perspective on survivorship disparities given its spread of urban and rural populations, racial and ethnic disparities and unique payor mix for patients (including provider-owned, nationwide and cooperative plans) [19]. Wisconsin has one NCI-designated comprehensive cancer center, 30 Commission on Cancer (CoC)-accredited cancer centers and an estimated 109 facilities that provide some degree of cancer care (some only offering chemotherapy or occasional visiting services). National data collection tends to capture patients diagnosed or treated at $\mathrm{CoC}$-accredited facilities, which are more likely to be located in larger urban areas [2,20]. Wisconsin also faces significant racial and ethnic health disparities. For example, Wisconsin has the nation's second largest BlackWhite disparity in lung cancer mortality and third largest Black-White disparity in female breast cancer mortality, with lower survival rates for Blacks compared to Whites for most cancers [21].

Here, we present the results of a survey developed to understand the availability and variability of cancer survivorship services throughout Wisconsin, which has a significant rural survivor demographic. To capture a more comprehensive understanding of services available in the state, our survey was not limited to $\mathrm{CoC}$-accredited facilities.

\section{Methods}

\section{Setting/participants}

The Wisconsin Cancer Collaborative (WCC) serves as the state's comprehensive cancer control program. The coalition is funded by the Centers for Disease Control (CDC) and is housed at the University of Wisconsin Carbone Cancer Center, in partnership with the Wisconsin Department of Health Services Division of Public Health. To assess the availability of cancer survivorship services in Wisconsin, the CDC funded the WCC to convene a survivorship advisory panel of experts to develop and disseminate a survey of cancer centers in Wisconsin.

\section{Survey development}

Study staff adapted the 41-item Patient-Centered Survivorship Care Index, which was originally developed as part of a Patient-Centered Outcomes Research Institute (PCORI) project (IH-12-11-5255) Evaluating Cancer Survivorship Care Models (see supplement one) [22]. Staff presented the survey to an advisory panel of multi-disciplinary cancer survivorship care experts, representing 16 different roles which serve cancer survivors. The panel included these roles: medical oncologists, hematologist, radiation oncologist, physical therapist, survivorship program nurse coordinator, psychologist, nurse/financial navigator, social work patient navigator, oncology nurse practitioner, oncology physician assistant, primary care provider (internist), Federally Qualified Health Center CEO, survivorship program manager, associate director of cancer prevention and control at a large cancer center, director of cancer survivorship and the program director of a local cancer survivorship community-based group. A cancer survivor's input was also included. Overall, 11 organizations were represented. The advisory panel pilot tested the survey to improve comprehension and add demographic questions, which staff incorporated into the final 33-question survey (see supplement two). Based on literature review, survivorship advisory panel feedback (provided via a Qualtrics survey and meeting), and review of relevant clinical guidelines, the index was edited to further breakdown the services being offered to cancer survivors and to better capture common late and long-term effects from cancer and its treatment. For example, clinical guidelines included in the original index provided guidance on common late and long-term effects. Panel feedback indicated that the original index was too broad, and a further breakdown of long- and late-term side 
effects was warranted to capturing the nuances of cancer survivorship services in Wisconsin. To further ensure that readers can understand the changes that we made, we have added the original index as supplement one and our adoption as supplement two. We aimed to provide an organizational assessment of cancer centers providing post-treatment cancer survivorship care services. Questions include topics such as: patient populations served, role of the person(s) providing oversight of the survivorship program, provider disciplines incorporated and not incorporated into survivorship care, services available to patients and caregivers and location of their provision, timing of discussion of various late/long-term effects occur, community partner(s) referral, and survivorship care plan provision. Survey instructions stated "for the purpose of this survey, survivorship care is care provided after cancer treatment is complete."

\section{Survey distribution}

A comprehensive list of cancer treatment facilities does not currently exist in Wisconsin. Study staff identified cancer treatment facilities by utilizing current accreditation lists, health system lists, and Google searches by county. Study staff identified and invited large health systems that indicated they provide cancer care to complete the survey via email. Outreach efforts aimed to ensure a representative sample of cancer treatment facilities in Wisconsin based on geographic spread and multiple health system participation. Rural-Urban Continuum Codes (RUCC) were utilized to classify cancer treatment facilities and centers by degree of rurality. RUCC data was obtained from the USDA and used to classify counties by their population size, degree of urbanization, and proximity to a metro area. After categorizing hospitals/cancer centers by RUCC code, a proposed site selection was created for equitable representation in rural areas (RUCC 4-9) and urban areas (RUCC 1-3). (See Fig. 1 below.)

Recruitment started in May 2020 and was conducted via existing communication channels including email, meetings with members of the Wisconsin Cancer Collaborative, and the e-newsletter. Survivorship advisory panel members and Wisconsin Cancer Collaborative members were also instrumental in identifying and contacting individuals for site recruitment. Given the COVID-19 pandemic, recruitment was extended from July to October 2020. Prospective sites were asked to indicate the following:

- 'We are interested in participating, please send right away.'

- 'We are interested in participating and can participate in June/July.'

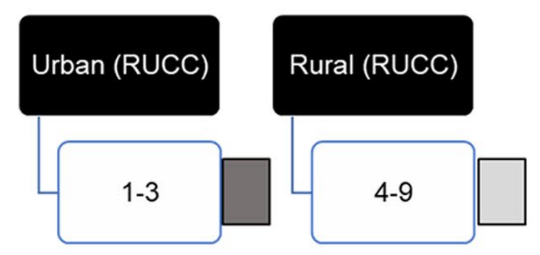

*Points mark locations of respondent sites

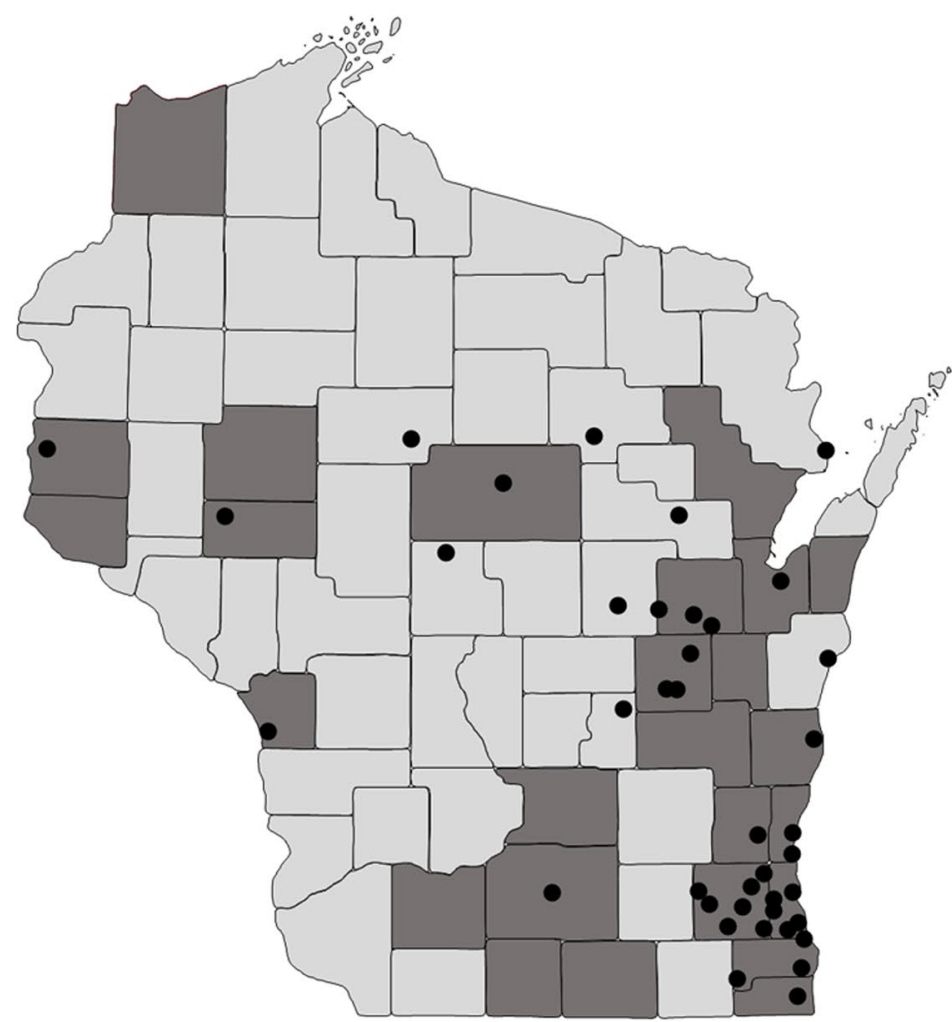

Fig. 1 Participating cancer centers/cancer treatment facilities plotted on Rural-Urban Continuum Codes (RUCC) map 
- 'We are interested in participating, but cannot participate until August/September.'

- 'We are not interested in participating.'

If sites indicated participating later on during the data collection period, we checked in again at a later time. We contacted sites up to six times, depending on their response. When receiving multiple non-responses, alternative contacts were identified for recruitment at that site.

The online survey was conducted via Qualtrics. Sites were asked to assemble a multi-disciplinary team of at least three to five individuals to conduct the survey. We suggested the team be comprised of an administrator, clinician, nurse, quality improvement specialist, and patient navigator. We strongly suggested including at least one provider responsible for conducting survivorship visits with patients. However, sites were allowed to determine the most suitable respondent to the survey, given structural differences across health systems. Due to the COVID-19 pandemic, recruitment became challenging as sites coped with reassignments, furloughs, and limited resources.

\section{Results}

\section{Respondents and characteristics}

Forty total sites out of 90 invited (44.4\% response rate) participated between June 2020 and October 2020; 32 sites were in RUCC 1-3 (urban), 8 sites in RUCC 4-9 (rural). Participating sites were mostly urban and clustered in two metropolitan areas. One site was an NCI-designated cancer center. 18 participating sites were $\mathrm{CoC}$-accredited. (Additionally, we found many satellite sites are not $\mathrm{CoC}$-accredited but may follow the same standards as the flagship site that is accredited.) The breakdown by patient population served by sites is as follows: Adult ages 18-100 (100\%; 40/40), Geriatric ages 65 years and above $(92.5 \% ; 37 / 40)$, AYA ages 15-39 (82.5\%; 33/40) and Pediatric ages 0-18 (7.5\%; 3/40). Many sites had a difficult time breaking down the number of patients per site, but often could report total number of patients for the health system. A majority of the health systems reported having 1000 or more patients. The highest reported total for a health system was 9235 patients. Four sites indicated smaller patient populations, ranging from 108 to 851 patients. Non-participating sites tended to be more rural and/or not offer any survivorship services.

\section{Services available}

The most common members of follow-up care teams were oncology physicians (medical oncology/hematology [100.0\%; 40/40], radiation [92.5\%; 37/40], and surgical
[87.5\%; 35/40]), nurse practitioners $(95.0 \% ; 38 / 40)$, and physician assistants $(87.5 \%$; 35/40). $100.0 \%$ of sites reported offering medical follow-up care (40/40), financial counseling (40/40) and dietetics (40/40). Over $90 \%$ of sites offered clinical trials $(97.5 \% ; 39 / 40)$, individual education during encounters $(97.5 \% ; 39 / 40)$, support groups $(95.0 \% ; 38 / 40)$, foreign language interpretation $(95.0 \% ; 38 / 40)$, spiritual support $(95.0 \% ; 38 / 40)$, and assistance with medication management $(92.5 \% ; 37 / 40)$. Other services were offered at moderate rates, including psychology or other behavioral health services $(67.5 \% ; 27 / 40)$, education for caregivers $(62.5 \% ; 25 / 40)$, and fertility specialists $(37.5 \% ; 15 / 40)$. Less than $50 \%$ of sites reported the following disciplines as part of their follow-up care team: reproductive endocrinology $(32.5 \%$; 13/40), patient navigation $(12.5 \%$; 5/40), community health workers $(10.0 \%$; $4 / 40)$, as defined by the American Society of Clinical Oncology as: "trusted individuals from local communities, who undergo training to support advocacy, community building and outreach, cultural competency, care coordination, and system navigation to promote health behavior change" [23], psychiatry $(5.0 \% ; 2 / 40)$, neuropsychology $(5.0 \% ; 2 / 40)$, and case management $(0.0 \%$; 0/40). (See Table 1, Table 2, and Table 3, for more details.)

Participants were asked to describe the degree to which various services were offered to each survivor treated at their facility. Dietary services $(42.5 \% ; 17 / 40)$, access to physical activity $(25.0 \% ; 10 / 40)$, and risk reduction services $(20.0 \%$; $8 / 40$ ) were rated as standards of care in less than half of sites. However, many sites reported working with community partners for these services, such as the Livestrong program and other programs associated with the YMCA $(92.5 \%$; 37/40). Maintaining a point of access with the survivor $(100.0 \% ; 40 / 40)$, as well clinician collaboration in addressing post-treatment needs $(92.5 \% ; 37 / 40)$ were most often rated as standards of care.

\section{Discussion of long-term side effects}

The survey assessed the frequency with which cancer treatment facilities discuss certain needs with survivors, as well as late/long-term effects of cancer treatment and diagnosis. A list of late/long-term effects of cancer treatment or diagnosis discussion is included in the survey (see Table 4). Discussion of late/long-term effects, in general, was most often rated as a standard of care $(100.0 \% ; 40 / 40)$, as was discussing the need for regular follow-up care and post-treatment screening $(97.5 \%$; 39/40). Receipt of a written treatment summary was also rated as a standard of care, but not as frequently as other services $(75.0 \%$; 30/40). Discussion on receiving emotional and social support was frequently rated as a standard of care $(85.0 \% ; 34 / 40)$, but referral to a specialist for mental/behavioral health concerns was not $(27.5 \%$; 11/40). Referral to a mental/behavioral health specialist 
Table 1 Responses to the question: "Which disciplines have not been integrated in follow-up care at your cancer center/cancer treatment facility?"

\begin{tabular}{lc}
\hline Discipline & $\%$ of Sites \\
\hline Community health worker & 72.5 \\
Reproductive endocrinologist & 67.5 \\
Psychiatrist & 65.0 \\
Case manager & 62.5 \\
Neuropsychologist & 52.5 \\
Gynecologist & 52.5 \\
Urologist & 45.0 \\
Clinical Nurse specialist & 40.0 \\
Psychologist & 32.5 \\
Behavioral Health counselor & 30.0 \\
Chaplain/Spiritual service provider & 27.5 \\
Patient navigator & 25.0 \\
Pharmacist & 25.0 \\
Survivorship coordinator & 20.0 \\
Radiation oncologist & 17.5 \\
Rehabilitation specialist & 17.5 \\
Social worker & 17.5 \\
Other & 15.0 \\
Nurse Navigator & 12.5 \\
Other medical specialist & 12.5 \\
Surgical oncologist & 10.0 \\
Financial Counselor & 7.5 \\
Nurse & 7.5 \\
Physician Assistant & 7.5 \\
Certified medical interpreter & 2.5 \\
Occupational therapist & 5.5 \\
Speech therapist & 5.0 \\
Palliative medicine provider & 5.0 \\
Primary care physician & 5.0 \\
Nurse Practitioner & 2.5 \\
Physical therapist & \\
Oncologist/Hematologist & \\
Nutritionist & 2.5 \\
\hline & \\
&
\end{tabular}

received the widest spread of responses; $37.5 \%$ (15/40) for provided at patient request, $27.5 \%$ (11/40) for standard of care, $17.5 \%$ (7/40) for referred elsewhere within the same organization, 15\% (6/40) for provided if patient met certain criteria, and $2.5 \%$ (1/40) for referred to another organization.

\section{Discussion}

Survivor quality of life can be influenced by many psychological, social, and physiological factors that result from cancer or cancer treatment. Unfortunately, our survey findings indicate many of the services addressing quality of life
Table 2 Responses to the question: "In your organization, what care team members currently provide follow-up care to survivors after treatment is complete?"

\begin{tabular}{lc}
\hline Discipline & \% of Sites \\
\hline Oncologist/Hematologist & 100.0 \\
Nurse Practitioner & 95.0 \\
Radiation oncologist & 92.5 \\
Surgical oncologist & 87.5 \\
Physician Assistant & 87.5 \\
Nurse Navigator & 85.0 \\
Physical therapist & 77.5 \\
Social worker & 65.0 \\
Speech therapist & 57.5 \\
Occupational therapist & 55.0 \\
Nutritionist & 55.0 \\
Primary care physician & 52.5 \\
Urologist & 52.5 \\
Gynecologist & 50.0 \\
Chaplain/ Spiritual service provider & 47.5 \\
Palliative medicine provider & 47.5 \\
Rehabilitation specialist & 42.5 \\
Pharmacist & 40.0 \\
Behavioral Health counselor & 37.5 \\
Psychologist & 35.0 \\
Reproductive endocrinologist & 3.0 \\
Survivorship coordinator & 0.0 \\
Financial Counselor & 0.0 \\
Certified medical interpreter & \\
Nurse & 27.5 \\
Clinical Nurse specialist & 27.5 \\
Patient navigator & 20.0 \\
Community health worker & 20.0 \\
Other medical specialist & 12.5 \\
Psychiatrist & 12.5 \\
Neuropsychologist & 10.0 \\
Case manager & 10.0 \\
\hline & \\
&
\end{tabular}

among survivors are not offered at most cancer treatment facilities in Wisconsin. While more research may be needed to understand reasons for these gaps, many of these services are not typically covered by insurance. Existing studies tend to address insurance's impact on cancer screening and care, but not survivorship service uptake and outcomes specifically. For example, Medicaid expansion increases access to insurance, which correlates with increased cancer screening utilization, and thus allows for earlier cancer detection [15]. Treatments are generally more effective at earlier stages, decreasing mortality particularly among patients with breast, colorectal, and lung cancers [24]. Analysis of the 2008-2010 Medical Expenditure Panel Survey found that 
Table 3 Responses to the question: "What survivorship services are available to patients through your cancer center/cancer treatment facility?"

\begin{tabular}{lc}
\hline Service & \% of Sites \\
\hline Medical follow-up care & 100.0 \\
Financial counseling/Navigation & 100.0 \\
Nutrition services/dietician & 100.0 \\
Individual education during encounters & 97.5 \\
Clinical trials & 97.5 \\
Support groups & 95.0 \\
Certified medical interpreter & 95.0 \\
Spiritual support services & 95.0 \\
Clinician education opportunities & 92.5 \\
Assistance with medication management & 92.5 \\
Genetic counseling & 82.5 \\
Virtual patient education opportunities & 80.0 \\
Exercise/fitness programs & 75.0 \\
Occupational rehabilitation/therapy & 75.0 \\
In-person classes opportunities & 72.5 \\
Physical rehabilitation & 72.5 \\
Speech rehabilitation & 72.5 \\
Smoking cessation services & 72.5 \\
Psychology/behavioral health services & 67.5 \\
Integrative medicine (e.g., yoga, acupuncture) & 67.5 \\
Palliative care/symptom management & 67.5 \\
Ostomy supplies/solutions & 65.0 \\
Sexual health services & 55.0 \\
Mastectomy prosthesis and bra fittings & 50.0 \\
Cognitive rehabilitation & 47.5 \\
Other & 42.5 \\
Psychiatry & 30.0 \\
Fertility services/specialists & 37.5 \\
Primary care transition services & 37.5 \\
Reproductive endocrinology & \\
Alcohol \& Other Drug Abuse services & \\
Workplace transition services & \\
\hline & \\
&
\end{tabular}

cancer survivors had equivalent or greater utilization of preventive care (an important component of survivorship care) compared to individuals without a history of cancer [14].

However, disparities in access to care for uninsured and publicly insured patients suggest that improvements in ability to access and utilize survivorship care are needed [14]. These findings highlight a need to address access to care issues and further encourage policies that ensure adequate insurance coverage to improve survivorship care outcomes and address survivors' needs.

Our statewide survey of health systems providing care for cancer survivors revealed gaps in mental health services, risk reduction services such as physical activity, and sexual health/fertility services. The Wisconsin Cancer Plan
2020-2030 calls for statewide and national policy considerations and strategies to improve coverage and access to many of the services identified as gap areas [25]. Reimbursement for many important survivorship services, such as behavioral interventions in survivors, is not currently available [26, 27]. The four recurrent underrepresented services that emerged in our survey were mental health services, exercise/lifestyle, sexual health, and fertility. Conversations with survey sites indicated lack of adequate insurance coverage of these services may be a unifying factor. Decision makers should base services on patient needs, rather than insurance coverage, by strongly advocating expanding and billing for services that are considered valuable for survivors and clinicians, like mental health resources. Additionally, we assessed both services provided and how often discussion of late/longterm effects were occurring. Discussion of late/long term effects does not necessarily equate to services being offered to help with that late/long term effect. However, a providerled discussion may help survivors further identify and utilize survivorship care options available to them. Discussions may be occurring, but lack of insurance coverage could limit provider referral or cancer survivors' utilization of a survivorship service. Furthermore, better integration of cancer survivorship guidelines, as well as increased concordance with those guidelines, among oncologists, specialists and primary care providers would help cancer survivors have more opportunities for their needs to be met. Adequate care of the cancer survivor does not solely fall on the oncologist. Primary care providers and specialists also play an increasingly important role in cancer survivorship and transitions of care should be improved.

Mental health services are a critical and often underutilized part of cancer survivorship, evident in our findings and existing literature [28]. Key barriers include lack of awareness and identification of mental health needs, lack of access to support services, physician time to screen, patients' lack of disclosure due to personal choice, and perceived stigma or perceived lack of effective treatment options [6]. Our survey supports this theme, as we found that less than half of survivorship follow-up care teams included mental health professionals and referral to mental health specialists was not a standard of care for many sites but referral for lateterm effects related to cancer care was. Considering cancer survivors show a significant level of distress (up to half in some studies), changes are necessary both at the policy and clinical level [5, 28]. Additionally, mental health services are often inadequately covered by insurance. Thus, our findings may speak to coverage-influenced underutilization even when cancer treatment facilities offer mental health services. A 2018 study by Perez et al. found that most childhood cancer survivors value having mental healthcare benefits; however, coverage and use of mental health services remain suboptimal [29]. Survivors of all ages experience 
Table 4 Responses to the questions: "Please indicate how often providers in your cancer center/cancer treatment facility typically discuss the following late/long-term effects with patients."

\begin{tabular}{|c|c|c|c|c|c|c|}
\hline & $\begin{array}{l}\text { Rarely or } \\
\text { never discuss } \\
(\%)\end{array}$ & $\begin{array}{l}\text { Discuss if patient } \\
\text { asks or reports } \\
\text { concerns }(\%)\end{array}$ & $\begin{array}{l}\text { Discuss with } \\
\text { patients most likely } \\
\text { to be at risk for this } \\
\text { effect }(\%)\end{array}$ & $\begin{array}{l}\text { Discuss with all or } \\
\text { almost all patients } \\
(\%)\end{array}$ & Don’t know (\%) & $\begin{array}{l}\text { Not applicable to our } \\
\text { patient population } \\
(\%)\end{array}$ \\
\hline Fatigue & 0.0 & 0.0 & 12.5 & 85.0 & 2.5 & 0.0 \\
\hline $\begin{array}{l}\text { Insomnia/Sleep } \\
\text { disorders }\end{array}$ & 0.0 & 10.0 & 60.0 & 22.5 & 7.5 & 0.0 \\
\hline $\begin{array}{l}\text { Health practice } \\
\text { changes (exercise, } \\
\text { smoking, diet, } \\
\text { alcohol consump- } \\
\text { tion) }\end{array}$ & 0.0 & 0.0 & 17.5 & 80.0 & 2.5 & 0.0 \\
\hline $\begin{array}{l}\text { Cognitive changes } \\
\text { (memory prob- } \\
\text { lems) }\end{array}$ & 0.0 & 2.5 & 67.5 & 30.0 & 0.0 & 0.0 \\
\hline Emotional distress & 0.0 & 7.5 & 0.0 & 90.0 & 2.5 & 0.0 \\
\hline $\begin{array}{l}\text { Mood changes } \\
\text { (depression, anxi- } \\
\text { ety, etc....) }\end{array}$ & 0.0 & 7.5 & 12.5 & 70.0 & 10.0 & 0.0 \\
\hline Digestive problems & 0.0 & 0.0 & 72.5 & 27.5 & 0.0 & 0.0 \\
\hline $\begin{array}{l}\text { Thyroid complica- } \\
\text { tions }\end{array}$ & 0.0 & 0.0 & 97.5 & 2.5 & 0.0 & 0.0 \\
\hline Dental problems & 0.0 & 2.5 & 92.5 & 2.5 & 0.0 & 2.5 \\
\hline Nerve damage & 0.0 & 0.0 & 80.0 & 20.0 & 0.0 & 0.0 \\
\hline Osteoporosis & 0.0 & 0.0 & 100.0 & 0.0 & 0.0 & 0.0 \\
\hline $\begin{array}{l}\text { Increased risk of } \\
\text { other cancers }\end{array}$ & 0.0 & 0.0 & 67.5 & 32.5 & 0.0 & 0.0 \\
\hline Sexual functioning & 7.5 & 10.0 & 65.0 & 17.5 & 0.0 & 0.0 \\
\hline Fertility & 0.0 & 2.5 & 97.5 & 0.0 & 0.0 & 0.0 \\
\hline $\begin{array}{l}\text { Ability to use the } \\
\text { bathroom without } \\
\text { difficulty }\end{array}$ & 0.0 & 25.0 & 70.0 & 2.5 & 2.5 & 0.0 \\
\hline Lymphedema & 0.0 & 0.0 & 92.5 & 7.5 & 0.0 & 0.0 \\
\hline Heart issues & 0.0 & 0.0 & 100.0 & 0.0 & 0.0 & 0.0 \\
\hline Lung issues & 0.0 & 0.0 & 100.0 & 0.0 & 0.0 & 0.0 \\
\hline
\end{tabular}

challenges accessing mental health care, especially younger cancer survivors and those that are uninsured or have a history of distress [29, 30]. Cancer survivors with serious psychological distress are less likely to stay up-to-date with important preventive services, which may negatively impact early identification of subsequent cancers [31]. Additionally, multiple respondent sites reported using National Comprehensive Cancer Network (NCCN) distress guidelines, which recommend distress screening for patients in all clinical settings [32]. While screening is necessary, measures to assure subsequent care are also needed. Moreover, our study found referral to a specialist for mental health concerns was not a standard of care, while discussion of emotional and social support was considered standard of care. Additionally, psychology services were included through only $67.5 \%$ (27/40) of sites, indicating a need for better integration. Even when mental health services are available, they are often expensive and may require long wait times. Increased insurance coverage of mental health services may aid prompt diagnosis and distress treatment among survivors.

Rehabilitation and exercise interventions help to improve cancer survivors' quality of life and survival by reducing the negative impact of long-term effects such as fatigue, pain or lymphedema, and are an important part of risk reduction for subsequent cancers and other comorbidities [33]. Guidelines commonly recognize and recommend rehabilitation as beneficial, but studies suggest that referral to these services and their integration into oncologic care is strikingly low [33]. Our findings indicate that only $25.0 \%$ (10/40) of respondent sites provide access to any physical activities for survivors as a standard of care. This could speak to a gap in insurance coverage or indicate well-established relationships 
with community partners. Coverage for rehabilitation services varies and exercise programs are usually referred out of the health system due to a lack of insurance coverage. Community organizations are essential in helping provide non-reimbursable services for cancer survivors. The Livestrong program at the YMCA, a community partner cited by several survey sites, has been found to improve physical (such as strength) and psychosocial measures (such as anxiety, fatigue, sleep disturbance, satisfaction with social roles) [34]. 32.5\% (13/40) of sites provide access to physical activity if requested by the patient. Guidelines advocate for, and survivors often value, tailored, supervised exercise interventions (for example by a physical therapist) and technology-based interventions, including activity trackers $[33,35,36]$. Improving exercise and rehabilitation guideline concordance could have substantial impact on quality of life among cancer survivors and should be prioritized, especially as the prevalence of cancer survivors increases [33].

Cancer and its treatment can negatively impact fertility [2]. Although this may not impact all patient populations at a cancer center, it is essential for cancer centers to assess if they need to tailor their programs and/or referrals to better meet their patients' needs. This is a particular concern for adolescent and young adult (AYA) survivors, who are often at higher risk for medical and psychosocial concerns and for whom fertility is likely to be a larger concern [37]. Reproductive endocrinology was included at about a third of follow-up care teams $(35.0 \% ; 14 / 40)$, as were fertility specialist services $(32.5 \% ; 13 / 40)$. Moreover, most sites that served AYA survivors in this survey did not have a reproductive endocrinologist on the care team and about half of sites that served AYA survivors offered fertility services/fertility specialists. This reinforces our discussion indicating that fertility and reproductive issues may be of concern but can be difficult for AYA survivors to discuss. Without those services, their demand and delivery are both difficult to measure and provide. Considering this, there is a documented need for fertility services due to cancer and other medical reasons. However, many fertility treatments are not considered medically necessary by insurance companies, and consequently are often not covered. Only 15 states currently require some private insurers to cover some fertility treatments [38]. Wisconsin does not require insurers to cover fertility treatments, and significant gaps in coverage exist. Out of pocket costs for fertility treatment may exceed $\$ 10,000$ depending on the service making this unattainable to many [38]. States with mandated in-vitro fertilization (IVF) insurance coverage appear to have higher IVF utilization (not cancer-specific), indicating that coverage may be linked to utilization of services and a potential need for policy solutions [38]. Additionally, patients may need to drive long distances due to limited service locations, limited resources as many people struggle with infertility, and long wait times, which is a complicating factor as cancer patients often cannot wait for these services. Further research is needed to assess if cancer survivors of childbearing age have timely and adequate access to fertility services within cancer facilities or the broader community.

Survivors also commonly report sexual dysfunction, which is often unaddressed, particularly among females [39, 40]. This lack of sexual health discussions occurs despite a desire for providers to address these issues [39-41]. Sexual health services were offered at $55.0 \%$ (22/40) of respondent sites and $50.0 \%$ (20/40) of sites provided gynecology follow-up care. Additionally, sexual functioning was the only late-term effect with any responses for "rarely or never discussed" $(7.5 \% ; 3 / 40)$, indicating a possible gap area. Insurance coverage and discussion of screening and treatment for sexual dysfunction should be incorporated into routine oncologic care [39].

Limitations to our study include that survey responses were self-reported from survey sites. This survey does not include every cancer treatment facility in Wisconsin. The study had a relatively low response rate (44\%) and only 8 of the 40 sites were considered rural (RUCC 4-9). Although attempts were made to ensure robust and representative participation, several sites and health systems either declined to participate or had to decline due to competing COVID19 priorities. Like many healthcare institutions across the USA during the COVID-19 pandemic, many staff at cancer treatment facilities faced staff shortages, furloughs, and reassignments which limited participation and may reflect the lower response rate, despite high initial interest. Additionally, lack of information about some cancer centers or lack of additional points of contact may have prevented participation for some sites. The implications of adapting the original Patient-Centered Survivorship Care Index should also be considered as a possible limitation. Utilizing an existing survey identified during literature review of cancer survivorship provided a starting instrument. As cancer survivorship is an evolving field, our adaptations allowed for more detailed measures of cancer survivorship services, especially considering unique rural considerations. However, these adaptations do not assess all survivorship services of assess cancer survivors' perspectives on these services. Additionally, comparability could be impacted if another state utilizes a different adaptation of the index. Comparability may also be influenced by Wisconsin's relatively high levels of health insurance coverage, employment, examples of racial disparities in cancer, and geographic accessibility of hospitals, relative to other states [42-44]. Wisconsin also has one NCI-designated cancer center which is comparable to the US ( 1.4 cancer centers per state) and the Midwest ( 0.95 cancer centers per state). Five different Midwest states have two NCI-designated cancer centers [45]. Additionally, Wisconsin ranks $17^{\text {th }}$ in number of $\mathrm{CoC}$-accredited facilities (30 total sites) in the USA [46]. Considering this, the mix of 
rural and urban communities within Wisconsin reflects both the generalizability of findings and limitations of comparing to more or less rural or urban states. Also, many cancer care locations do not provide any cancer survivorship care. This is consequent to an increased focus on acute cancer treatment at these locations such as chemotherapy administration, especially in more rural areas. Moreover, cancer survivorship services are limited in rural areas, and rural site participation was lower. This highlights an important opportunity for survivorship program expansion in Wisconsin. Survivorship care services are often found at the health system's main site, thus many health systems felt it inappropriate for their other outreach sites to participate. Additionally, not all participating sites were able to report characteristics such as number of patients served. Further collection of additional characteristics of cancer treatment facilities (such as type of facility) would be beneficial to future research.

\section{Conclusion}

The results of this survey identified strengths and gaps in services that cancer treatment facilities provide. This study serves as a concrete example of the potential for growth in preventive and psychological care within all medical settings. Additionally, many of the gaps in services identified in this survey may be linked to insurance coverage, indicating a need for policy considerations such as increased insurance coverage for essential survivorship services. Gaps in service include mental health services, risk reduction services such as physical activity, and sexual health/fertility services. Services that address the physiological and psychological effects of cancer treatment are essential to the health and well-being of cancer survivors and require increased coverage. These services have been shown to improve pain, functioning and overall quality of life throughout the entire stage of survivorship [47]. It is essential for healthcare providers and health systems to understand and address the unique medical and psychosocial needs of survivors, but it is also important for these services to be adequately covered by insurance. Future research should look to understand health screening practices, as opposed to referral standards, and understanding which services cancer centers view as most needed, given the context of their location and patient population.

Supplementary Information The online version contains supplementary material available at https://doi.org/10.1007/s11764-021-01117-4.

Acknowledgements Our survey was adapted from the 41-item PatientCentered Survivorship Care Index that was originally developed as part of a Patient-Centered Outcomes Research Institute (PCORI) project (IH-12-11-5255) Evaluating Cancer Survivorship Care Models.
Thank you to Dr. Cibele Barbosa Carroll, the UWCCC Survivorship Program, and Allison Antoine for their review.

We want to thank the cancer centers and cancer treatment facilities that participated in this survey and subsequent survivorship community of practice. We also want to thank the individuals that served on the survivorship advisory panel which aided in the creation of this survey.

Author contribution All authors contributed to the study conception and design. Material preparation, data collection and analysis were performed by Alexandria Cull Weatherer and John Krebsbach. The first draft of the manuscript was written by Alexandria Cull Weatherer and John Krebsbach and all authors commented on previous versions of the manuscript. All authors read and approved the final manuscript.

Funding Centers for Disease Control and Prevention (grant 5NU58DP006328-04-00); Core grant University of Wisconsin Carbone Cancer Center Support Grant P30 CA014520;1701 survivorship supplement to NCCCP award (Grant 144 AAI1883 5).

Data availability The datasets generated during and/or analyzed during the current study are available from the corresponding author on reasonable request in aggregate form.

Code availability Not applicable.

\section{Declarations}

Ethics approval and consent to participate The University of Wisconsin- Madison IRB has confirmed that no ethical approval is required, and this study was exempt. This study was exempt from the IRB. All survey sites consented to participation when filling out the survey in Qualtrics. Participation was voluntary and able to be rescinded at any time.

Consent for publication All survey sites agreed to responses being shared in an aggregate from for publication when they filled out the survey.

Conflict of interest This manuscript is not being considered elsewhere. The study results have only been discussed with survey sites as of now. All the authors declare no financial or non-financial conflicts of interest. There are no disclaimers.

\section{References}

1. National Cancer Institute: Office of Cancer Survivorship. 2020

2. Miller KD, Nogueira L, Mariotto AB, et al. Cancer treatment and survivorship statistics, 2019. CA Cancer J Clin. 2019;69:363-85. https://doi.org/10.3322/caac.21565.

3. Bluethmann SM, Mariotto AB, Rowland JH. Anticipating the "silver tsunami": prevalence trajectories and comorbidity burden among older cancer survivors in the United States. Cancer Epidemiol Biomarkers Prev. 2016;25:1029-36. https://doi.org/10.1158/ 1055-9965.EPI-16-0133.

4. de Moor JS, Mariotto AB, Parry C, et al. Cancer survivors in the United States: prevalence across the survivorship trajectory and implications for care. Cancer Epidemiol Biomarkers Prev. 2013;22:561-70. https://doi.org/10.1158/1055-9965.EPI-12-1356.

5. Hoffman KE, McCarthy EP, Recklitis CJ, et al. Psychological distress in long-term survivors of adult-onset cancer: results from a national survey. Arch Intern Med. 2009;169:1274-81. https:// doi.org/10.1001/archinternmed.2009.179. 
6. Niedzwiedz CL, Knifton L, Robb KA, et al. Depression and anxiety among people living with and beyond cancer: a growing clinical and research priority. BMC Cancer. 2019;19:943. https://doi.org/10.1186/s12885-019-6181-4.

7. Yang W, Williams JH, Hogan PF, et al. Projected supply of and demand for oncologists and radiation oncologists through 2025: an aging, better-insured population will result in shortage. J Oncol Pract. 2014;10:39-45. https://doi.org/10.1200/JOP.2013. 001319.

8. Mayer DK, Nasso SF, Earp JA. Defining cancer survivors, their needs, and perspectives on survivorship health care in the USA. Lancet Oncol. 2017;18:e11-8. https://doi.org/10.1016/S14702045(16)30573-3.

9. American Society of Clinical Oncology: Institute of Medicine. From cancer patient to cancer survivor: lost in transition, The National Academies Press. 2005

10. Blaes AH, Adamson PC, Foxhall L, et al. Survivorship care plans and the commission on cancer standards: the increasing need for better strategies to improve the outcome for survivors of cancer. JCO Oncol Pract. 2020;16:447-50. https://doi.org/10.1200/JOP. 19.00801 .

11. Halpern MT, Viswanathan M, Evans TS, et al. Models of cancer survivorship care: overview and summary of current evidence. J Oncol Pract. 2015;11:e19-27. https://doi.org/10.1200/JOP.2014. 001403.

12. Argenbright KE, Berry E, Mazour T, et al. Cancer survivorship care in the rural community: a mobile model, American Society of Clinical Oncology, 2016. J Clin Oncol. 2016;34(3):38-38. https:// doi.org/10.1200/jco.2016.34.3_suppl.38.

13. Yabroff KR, Short PF, Machlin S, et al. Access to preventive health care for cancer survivors. Am J Prev Med. 2013;45:304-12. https://doi.org/10.1016/j.amepre.2013.04.021.

14. Bellizzi KM, Aziz NM, Rowland JH, et al. Double jeopardy? Age, race, and HRQOL in older adults with cancer. J Cancer Epidemiol. 2012;2012:478642. https://doi.org/10.1155/2012/478642.

15. Yabroff KR, Reeder-Hayes K, Zhao J, et al. Health insurance coverage disruptions and cancer care and outcomes: systematic review of published research. J Natl Cancer Inst. 2020;112:67187. https://doi.org/10.1093/jnci/djaa048.

16. Weaver KE, Palmer N, Lu L, et al. Rural-urban differences in health behaviors and implications for health status among US cancer survivors. Cancer Causes Control. 2013;24:1481-90. https:// doi.org/10.1007/s10552-013-0225-x.

17. Levit LA, Byatt L, Lyss AP, et al. Closing the rural cancer care gap: three institutional approaches. JCO Oncol Pract. 2020;16:422-30. https://doi.org/10.1200/OP.20.00174.

18. Nipp R, Tramontano AC, Kong CY, et al. Disparities in cancer outcomes across age, sex, and race/ethnicity among patients with pancreatic cancer. Cancer Med. 2018;7:525-35. https://doi.org/ 10.1002/cam4.1277.

19. Office of the Comissioner of Insurance: Map of Comprehensive Health Insurers - Individual Market, 2020, pp https://oci.wi.gov/ Pages/Consumers/FindHealthInsurer.aspx

20. Bilimoria KY, Bentrem DJ, Stewart AK, et al. Comparison of commission on cancer-approved and -nonapproved hospitals in the United States: implications for studies that use the National Cancer Data Base. J Clin Oncol. 2009;27:4177-81. https://doi. org/10.1200/JCO.2008.21.7018.

21. Olson J, Cawthra T, Beyer K, et al. Community and research perspectives on cancer disparities in Wisconsin. Prev Chronic Dis. 2020;17:E122. https://doi.org/10.5888/pcd17.200183.

22. Mead KHRS, Arem H, et al. Evaluating Different Types of Cancer Survivorship Care. Washington, DC: Patient-Centered Outcomes Research Institute (PCORI); 2019.

23 Sedhom R, et al. Can community health workers increase palliative care use for African American patients? A pilot study. JCO
Oncology Practice. 2021;17(2):e158-67. https://doi.org/10.1200/ OP.20.00574.

24. Lam MB, Phelan J, Orav EJ, et al. Medicaid expansion and mortality among patients with breast, lung, and colorectal cancer. JAMA Netw Open. 2020;3:e2024366. https://doi.org/10.1001/ jamanetworkopen.2020.24366.

25. University of Wisconsin Carbone Cancer Center and Wisconsin Department of Health Services; Wisconsin Cancer Plan 2020 2030. Madison, WI: 2020. Available online at: www.wicancer.org.

26. (ASCO) ASoCO: Coverage \& Reimbursement for Survivorship Care Services

27. Kline RM, Arora NK, Bradley CJ, et al. Long-term survivorship care after cancer treatment - summary of a 2017 National Cancer Policy Forum Workshop. J Natl Cancer Inst. 2018;110:1300-10. https://doi.org/10.1093/jnci/djy176.

28. Mehnert A, Hartung TJ, Friedrich M, et al. One in two cancer patients is significantly distressed: prevalece and indicators of distress. Psychooncology. 2018;27:75-82. https://doi.org/10.1002/ pon.4464.

29. Perez GK, Kirchhoff AC, Recklitis C, et al. Mental health insurance access and utilization among childhood cancer survivors: a report from the childhood cancer survivor study. J Cancer Surviv. 2018;12:528-36. https://doi.org/10.1007/s11764-018-0691-y.

30. Li C, Forsythe L, Lerro C, et al. Mental health services utilization and expenditures associated with cancer survivorship in the United States. J Cancer Surviv. 2015;9:50-8. https://doi.org/10. 1007/s11764-014-0392-0.

31. Rim SH, Yabroff KR, Dasari S, et al. Preventive care service use among cancer survivors with serious psychological distress: an analysis of the medical expenditure panel survey data. Prev Med. 2019;123:152-9. https://doi.org/10.1016/j.ypmed.2019.03.024.

32. National Comprehensive Cancer Network: NCCN Clinical Practice Guidelines in Oncology Distress Management Version 2.2021. 2021

33. Stout NL, Santa Mina D, Lyons KD, et al. A systematic review of rehabilitation and exercise recommendations in oncology guidelines. CA: A Cancer Journal for Clinicians. 2020. https://doi.org/ 10.3322/caac. 21639

34. Schumacher MM, McNiel P. the impact of Livestrong ${ }^{\circledR}$ at the YMCA for cancer survivors. Oncol Nurs Forum. 2018;45:717-25. https://doi.org/10.1188/18.ONF.717-725.

35. Ten Tusscher MR, Groen WG, Geleijn E, et al. Physical problems, functional limitations, and preferences for physical therapistguided exercise programs among Dutch patients with metastatic breast cancer: a mixed methods study. Support Care Cancer. 2019;27:3061-70. https://doi.org/10.1007/s00520-018-4619-x.

36. Cadmus-Bertram L, Tevaarwerk AJ, Sesto ME, et al. Building a physical activity intervention into clinical care for breast and colorectal cancer survivors in Wisconsin: a randomized controlled pilot trial. J Cancer Surviv. 2019;13:593-602. https://doi.org/10. 1007/s11764-019-00778-6.

37. Hydeman JA, Uwazurike OC, Adeyemi EI, et al. Survivorship needs of adolescent and young adult cancer survivors: a concept mapping analysis. J Cancer Surviv. 2019;13:34-42. https://doi. org/10.1007/s11764-018-0725-5.

38. Weigel G, Ranji U, Long M, et al. Coverage and use of fertility services in the U.S., Kaiser Family Foundation. 2020

39. Falk SJ, Dizon DS. Sexual health issues in cancer survivors. Semin Oncol Nurs. 2020;36: 150981. https://doi.org/10.1016/j. soncn.2019.150981.

40. Taylor J, Ruggiero M, Maity A, et al. Sexual health often overlooked in cancer survivorship care, especially for female patients, American Society for Radiation Oncology (ASTRO). 2020

41. Frederick NN, Recklitis CJ, Blackmon JE, et al. Sexual dysfunction in young adult survivors of childhood cancer. Pediatric Blood Cancer. 2016;63:1622-8. https://doi.org/10.1002/pbc.26041. 
42. Kaiser Family Foundation: State Health Facts. Health Insurance Coverage of the Total Population, 2019. Accessed September 17, 2021. https://www.kff.org/other/state-indicator/total-population/? currentTimeframe $=0 \&$ sortModel $=\% 7 \mathrm{~B} \% 22$ colId $\% 22: \% 22 \mathrm{Loc}$ ation $\% 22, \% 22$ sort $\% 22: \% 22$ asc $\% 22 \% 7 \mathrm{D}$

43. U.S. Bureau of Labor Statistics: Local Area Unemployment Statistics. Unemployment Rate for States, Seasonally Adjusted. 2021

44. Black, P. Defining rural for Wisconsin. Wisconsin Office of Rural Health. 2016

45. National Cancer Institute: Find a Cancer Center. 2021. Accessed September 17, 2021. https://www.cancer.gov/research/infrastruc ture/cancer-centers/find

46. American College of Surgeons: searching for accredited cancer programs. 2021. Accessed September 17, 2021. https://www.facs. org/search/cancer-programs
47. Silver JK, Baima J, Mayer RS. Impairment-driven cancer rehabilitation: an essential component of quality care and survivorship. CA Cancer J Clin. 2013;63:295-317. https://doi.org/10.3322/caac. 21186.

Publisher's note Springer Nature remains neutral with regard to jurisdictional claims in published maps and institutional affiliations. 